\title{
Stressful life events and serum triglyceride levels: the Cardiovascular and Metabolic Diseases Etiology Research Center cohort in Korea
}

\author{
Naharin Sultana Anni', Sun Jae Jung ${ }^{1,2}$, Jee-Seon Shim ${ }^{1,3}$, Yong Woo Jeon', Ga Bin Lee', \\ Hyeon Chang Kim ${ }^{1,2,3}$ \\ 'Department of Public Health, Graduate School, Yonsei University, Seoul, Korea; ${ }^{2}$ Department of Preventive Medicine, Yonsei University College \\ of Medicine, Seoul, Korea; ${ }^{3}$ Cardiovascular and Metabolic Diseases Etiology Research Center, Yonsei University College of Medicine, Seoul, Korea
}

\begin{abstract}
OBJECTIVES: Elevated serum triglyceride levels are a risk factor for developing cardiovascular disease. A number of studies have demonstrated a positive association between psychological stress and serum triglyceride levels. However, there is limited evidence regarding the impact of stressful life events (SLEs) on serum triglyceride levels in the healthy population. Therefore, we evaluated the independent association between SLEs and serum triglyceride levels in a middle-aged Korean population.

METHODS: We analyzed a sample of 2,963 people (aged 30-64 years; 36\% men) using baseline data from the Cardiovascular and Metabolic Diseases Etiology Research Center (CMERC) cohort study. The Korean version of the Life Experience Survey questionnaire was used to measure the presence and positive/negative impact of SLEs. Hypertriglyceridemia was defined as a fasting serum triglyceride level of $\geq 150 \mathrm{mg} / \mathrm{dL}$.

RESULTS: Of the 2,963 participants, 33.1\% reported at least 1 SLE over the past 6 months and 24.8\% had hypertriglyceridemia. Even after adjusting for potential confounders, the serum triglyceride level was significantly associated with the total number of SLEs in men $(3.333 \mathrm{mg} / \mathrm{dL}$ per event; $\mathrm{p}=0.001)$, but not in women $(0.451 \mathrm{mg} / \mathrm{dL}$ per event, $\mathrm{p}=0.338)$. Hypertriglyceridemia was also associated with having 4 or more SLEs with positive effects (odds ratio [OR], 2.57; 95\% CI, 1.02 to 6.46) and 4 or more SLEs with negative effects (OR, 1.99; 95\% CI, 1.16 to 3.41 ) in men.
\end{abstract}

CONCLUSIONS: Our findings suggest that SLEs may increase the risk of hypertriglyceridemia in middle-aged men.

KEY WORDS: Psychological stress, Lipids, Hypertriglyceridemia

\section{INTRODUCTION}

Dyslipidemia, which consists of increased blood total cholesterol, low-density lipoprotein (LDL)-cholesterol, and triglyceride

\section{Correspondence: Hyeon Chang Kim}

Department of Preventive Medicine, Yonsei University College of Medicine, 50-1 Yonsei-ro, Seodaemun-gu, Seoul 03722, Korea E-mail:hckim@yuhs.ac

Received: Nov 13, 2020 / Accepted: Jun 9, 2021 / Published: Jun 9, 2021

This article is available from: https://e-epih.org/

(c) This is an open-access article distributed under the terms of the Creative Commons Attribution License (https://creativecommons.org/licenses/by/4.0/), which permits unrestricted use, distribution, and reproduction in any medium, provided the original work is properly cited.

(C) 2021, Korean Society of Epidemiology levels and decreased high-density lipoprotein (HDL)-cholesterol levels, is one of the most strongly contributory risk factors for cardiovascular disease. High triglyceride levels are now considered an independent risk factor of cardiovascular disease [1]. Several studies have reported that a high serum triglyceride level alone or in combination with a low HDL-cholesterol or high LDL-cholesterol level is associated with atherosclerotic cardiovascular disease $[2,3]$.

There is increasing evidence suggesting that physical and psychological stress can affect human physiological parameters, including the lipid profile [4], and increase the risk of cardiovascular disease and mortality [5]. It is hypothesized that the activity of the hypothalamic-pituitary-adrenal (HPA) axis can be increased due to chronic stress, and the resultant increase in cortisol levels leads to an upsurge in both insulin resistance and abdominal obe- 
sity with lipids and blood pressure, thereby facilitating the development of metabolic syndrome [6]. Additionally, physical stress generated through physical activity can also affect the lipid profile [7]. The workplace is an important source of both mental stress and physical stress. Several studies have been conducted among specific types of workers to evaluate the effect of stress or stressful events in the workplace on metabolic abnormalities [8,9]. Although the findings of those studies strongly support the adverse metabolic effects of job-related stress, those findings cannot be generalized to stress in other situations. There is still scarce evidence for independent associations between psychological stress from life events and the lipid profile.

The Korean population is known to have a high level of psychological stress and an exceptionally high rate of suicide. A recent Korean cohort study, involving 1,071 participants aged 15-16 years who were followed up for an average of 6 years, reported that consistently low or increased total cholesterol levels during adolescence predicted an elevated risk of depressive symptoms in early adulthood [10]. However, the effects of life-related stress on the blood lipid profile have not been investigated previously in the healthy Korean population. Therefore, we evaluated the association between stressful life events (SLEs) and serum triglyceride levels, as a component of the lipid profile, in a middle-aged Korean population.

\section{MATERIALS AND METHODS}

\section{Study population}

The Cardiovascular and Metabolic Diseases Etiology Research Center (CMERC) cohort data were used for this study. The recruitment and baseline examinations of the CMERC community cohort were performed from 2013 to 2018 at 2 study centers: center 1 was located in Seoul, and center 2 was located in Suwon, Gyeonggi Province [11]. Participants were recruited mostly through advertisements in regional newspapers, promotional posters in public areas, or through the acquaintances of study participants. Data from center 1 were selected for the present study because of accessibility. The participants from center 1 were residents of Seoul and its western suburbs, tended to be more educated, were less likely to be married, and had higher blood lipid levels than those from center 2. Nevertheless, there were no significant differences in SLEs between the 2 centers (Supplementary Material 1). A total of 4,060 men and women aged 30-64 years without a history of myocardial infarction, heart failure, stroke, or cancer completed examinations at center 1 . After excluding those who had missing data for the study variables $(n=495)$, lipid-modifying drug users $(n=461)$, and patients diagnosed with diabetes $(n=216)$ or kidney disease $(n=7), 2,963$ people were left, yielding the sample of the present study (Supplementary Material 2).

\section{Measurements}

In the CMERC cohort, trained interviewers examined subjects using the Life Experiences Survey Questionnaire [12], which has been translated to Korean and validated for the Korean population [13]. Participants were asked about a total of 50 SLEs, including marital status, death of a close family member, sexual difficulties, or conflict with colleagues, that occurred any time during the last 6 months of the participants' life. The distribution of 50 types of SLEs by frequency (yes or no) and their impact (positive impact, no impact, and negative impact) are provided in Supplementary Material 3. The participants were instructed to mark whether they experienced those life event items and were given a 7-point scale ranging from +3 (extremely positive effect) to -3 (extremely negative effect). In our study, we calculated the total number of SLEs, the number of SLEs with positive effects, and the number of SLEs with negative effects, and then divided the numbers into 4 groups (no, 1, 2-3, and $\geq 4$ events). Furthermore, we considered the SLE scores as a measure of the direction and strength of each event. We calculated the sums of positive or negative effect scores and divided them into 4 groups similar to those calculated for events (scores of $0,1,2-3$, and $\geq 4$ ).

Serum total cholesterol, HDL-cholesterol, LDL-cholesterol, and triglyceride levels were measured by an auto-analyzer (ADVIA 1800 Auto Analyzer; Siemens Medical Solutions, Malvern, PA, USA) from blood samples that were collected after at least 8 hours of fasting. All laboratory tests were performed in a central laboratory (Seoul Clinical Laboratories R\&D Center, Seoul, Korea). Hypertriglyceridemia was defined as a serum triglyceride level $\geq 150 \mathrm{mg} / \mathrm{dL}$ [14]. Hypo-HDL-cholesterolemia was defined as an HDL-cholesterol level $<40 \mathrm{mg} / \mathrm{dL}$ and hyper-LDL-cholesterolemia was defined as an LDL-cholesterol level $\geq 160 \mathrm{mg} / \mathrm{dL}$ [15].

Socio-demographic information, medical history, and healthrelated behaviors were collected by trained interviewers following a standardized protocol in systematic face-to-face interviews. Socio-demographic factors included age, gender, education level (low: completion of secondary school or below; middle: completion of high school; high: completion of university or more), household income (divided into quartiles), and marriage (married or unmarried). Height was measured using a stadiometer (DS-102 Jenix; Seoul, Korea) to the nearest $0.1 \mathrm{~cm}$, and weight was measured using a digital scale (DB-150; CAS, Seongnam, Korea) to the nearest $0.1 \mathrm{~kg}$. Body mass index (BMI) was calculated as weight $(\mathrm{kg})$ divided by height squared $\left(\mathrm{m}^{2}\right)$. Waist circumference was measured at the middle point between the lower border of the rib cage and iliac crest. Blood pressure was recorded thrice after 5 minutes of rest in the sitting position using an automated oscillometric device placed on the right arm (HEM-7080; Omron Health, Matsusaka, Japan); the mean of 2 values (second and third) was calculated. Health-related behaviors included smoking status (never smokers, who smoked $<100$ cigarettes throughout their lifetime; past smokers; and current smokers), alcohol consumption (non-drinkers, past drinkers, and current drinkers) [16], physical activity, and sleep duration. Physical activity was assessed using the Korean version of the International Physical Activity Questionnaire (IPAQ)-short form [17] where the last 7 days of activities were recorded and participants were 
divided into 3 groups (low, moderate, and high) following the IPAQ guidelines [18]. Sleep duration was self-reported and grouped into quartiles ( $<6.8,6.8-7.7,7.8-8.7$, and $\geq 8.8$ hours). The Beck Depression Inventory II (BDI-II), which has been validated for the Korean population, was used to measure depressive symptoms [19]. The BDI-II consists of 21 items measured on a Likert scale from 0 to 3 , with questions on symptoms related to depression in the previous 2 weeks. Higher scores represent more severe depressive symptoms. Nutritional information was collected using a semi-quantitative food frequency questionnaire, which was developed and validated for assessing Korean adults in the Korean National Health and Nutrition Examination Survey [20]. The intake of major macronutrients (total calories, carbohydrates, protein, and fat) was calculated.

\section{Statistical analysis}

Since it is known that men and women have different stress responses and blood lipid distributions, all statistical analyses were performed separately for men and women. It has also been reported that the distribution of dyslipidemia among Koreans differs not only by gender, but also by menopausal status [21]. Thus, the analysis for women was further stratified into premenopausal women and postmenopausal women, as presented in Supplementary Material 4. To identify differences in general characteristics in the study population stratified by gender, the independent $\mathrm{t}$ test for normally distributed continuous variables, the chi-square test for categorical variables, and the Mann-Whitney U test for continuous variables with a skewed distribution were performed. Data are presented as mean with standard deviation, median with interquartile range, or number with percentage. Multiple linear regression models were computed to examine the associations between SLEs and each category of serum lipid levels. Adjustment was performed for potential confounders, such as age, BMI, waist circumference, systolic blood pressure, diastolic blood pressure, education level, marital status, smoking, drinking, physical activity, dietary (total energy, carbohydrate, and fat) intake, and depression score. Furthermore, binary logistic regression analysis was performed along with adjustment for all potential confounders to compare serum triglyceride levels according to the number of SLEs. Statistical analyses were conducted using SAS version 9.4 (SAS Institute Inc., Cary, NC, USA), and statistical significance was set at $\mathrm{p}$-value $<0.05$.

\section{Ethics statement}

This study was approved by the Institutional Review Board of Yonsei University Health System, Seoul, Korea (4-2013-0661). Before participating in the study, all participants provided written informed consent.

\section{RESULTS}

The characteristics of all study participants $(n=2,963)$ divided by gender (1,076 men and 1,887 women) are presented in Table 1 .
The mean age of all participants was $50.2 \pm 9.7$ years. The median (interquartile range) number of total SLEs was 3 (1-5), whereas the median (interquartile range) number of SLEs with positive and negative effects was 1 (1-2) and 1 (1-3), respectively. No significant difference was found in the median number of SLEs with positive and negative effects between men and women. Hypertriglyceridemia was found in $24.8 \%$ of all participants. Men participants had a higher prevalence of hypertriglyceridemia (38.4\%) than women participants (17.0\%) (data not shown). The mean serum triglyceride level was $127.0 \pm 86.9 \mathrm{mg} / \mathrm{dL}$ for all participants. The mean serum triglyceride level was significantly higher in men $(156.0 \pm 111.3 \mathrm{mg} / \mathrm{dL})$ than in women $(110.5 \pm 63.8 \mathrm{mg} / \mathrm{dL})$.

\section{Stressful life events and lipid levels}

The association between different types of SLEs (including SLEs with positive effects and SLEs with negative effects) and lipid levels are presented in Table 2. We found a positive association between SLEs with negative effects (odds ratio [OR], 1.29; 95\% confidence interval [CI], 1.07 to 1.72 ) and hypertriglyceridemia in men. However, a significant association was found for SLEs with positive effects (OR, 1.47; 95\% CI, 1.14 to 1.89) in women. Furthermore, we did not find a significant association between different types of SLEs and hypo-HDL-cholesterolemia or hyper-LDL-cholesterolemia in either men or women. Table 3 presents the risk of hypertriglyceridemia according to the number of total SLEs, SLEs with positive effects, SLEs with negative effects, and their sum of scores in men and women, respectively. Men who had $\geq 4$ SLEs with positive effects had a higher likelihood of having hypertriglyceridemia (OR, 2.57; 95\% CI, 1.02 to 6.46) than those who had no SLEs in the past 6 months. Similar results were also observed for SLEs with negative effects after adjusting for potential confounders (OR, 1.99; 95\% CI, 1.16 to 3.41). Following the same trend, an increased OR also was seen for the sum of the negative effect score (OR, 1.71; 95\% CI, 1.11 to 2.55$)$ in men. In addition, hypertriglyceridemia was associated with a per-event increase in the total number of SLEs (OR, $1.05 ; 95 \% \mathrm{CI}, 1.01$ to 1.09 ), the number of SLEs with negative effects (OR, 1.07; 95\% CI, 1.01 to 1.13), and the sum of the negative effect score (OR, 1.04; 95\% CI, 1.01 to 1.08 ) (Table 3). However, no significant association was observed in women (Table 3), either pre-menopausal or post-menopausal (Supplementary Material 4).

Multiple linear regression analysis of male participants showed that number of SLEs had a positive association with serum triglyceride levels. After adjusting for all confounders in a model, the total number of SLEs $(\beta=3.333 ; p=0.001)$, SLEs with positive effects $(\beta=5.557 ; p=0.007)$, the sum of positive affect score $(\beta=2.229$; $\mathrm{p}=0.041)$, and SLEs with negative effects $(\beta=3.286 ; \mathrm{p}=0.021)$ showed positive linear associations with serum triglyceride levels (Table 4). 
Table 1. General characteristics of the total study population and according to gender

\begin{tabular}{|c|c|c|c|c|}
\hline Variables & Total $(n=2,963)$ & Men $(n=1,076)$ & Women $(n=1,887)$ & p-value ${ }^{1}$ \\
\hline Age (yr) & $50.2 \pm 9.7$ & $49.1 \pm 10.5$ & $50.8 \pm 9.2$ & $<0.001$ \\
\hline Body mass index $\left(\mathrm{kg} / \mathrm{m}^{2}\right)$ & $23.8 \pm 3.1$ & $24.8 \pm 2.9$ & $23.1 \pm 2.9$ & $<0.001$ \\
\hline Waist circumference $(\mathrm{cm})$ & $81.3 \pm 9.2$ & $81.6 \pm 9.5$ & $81.1 \pm 9.1$ & 0.112 \\
\hline Systolic blood pressure $(\mathrm{mmHg})$ & $118.5 \pm 15.1$ & $125.4 \pm 13.8$ & $114.5 \pm 14.4$ & $<0.001$ \\
\hline Diastolic blood pressure $(\mathrm{mmHg})$ & $76.4 \pm 10.2$ & $81.1 \pm 10.2$ & $73.7 \pm 9.3$ & $<0.001$ \\
\hline \multicolumn{5}{|l|}{ Education } \\
\hline$\leq$ Middle school or less & $334(11.3)$ & $81(7.5)$ & $253(13.4)$ & \multirow[t]{3}{*}{$<0.001$} \\
\hline High school & $1141(38.5)$ & $309(28.7)$ & $832(44.1)$ & \\
\hline$\geq$ University or more & $1,488(50.2)$ & $686(63.8)$ & $802(42.5)$ & \\
\hline \multicolumn{5}{|l|}{ Household income (yr) } \\
\hline Q1 & $701(23.7)$ & $212(19.7)$ & $489(25.9)$ & \multirow[t]{4}{*}{$<0.001$} \\
\hline Q2 & $549(18.5)$ & $204(19.0)$ & $345(18.3)$ & \\
\hline Q3 & $926(31.3)$ & $347(32.2)$ & $579(30.7)$ & \\
\hline Q4 & $787(26.6)$ & $313(29.1)$ & $474(25.1)$ & \\
\hline Married & $2,768(93.4)$ & $986(91.6)$ & $1,782(94.4)$ & 0.004 \\
\hline \multicolumn{5}{|l|}{ Smoking } \\
\hline Never smoker & $2,006(67.7)$ & $254(23.6)$ & $1,752(92.8)$ & \multirow[t]{3}{*}{$<0.001$} \\
\hline Past smoker & $523(17.7)$ & $448(41.6)$ & $75(4.0)$ & \\
\hline Current smoker & $434(14.6)$ & $374(34.8)$ & $60(3.2)$ & \\
\hline \multicolumn{5}{|l|}{ Drinking } \\
\hline Non-drinker & $564(19.0)$ & $76(7.1)$ & $488(25.9)$ & \multirow[t]{3}{*}{$<0.001$} \\
\hline Past drinker & $135(4.6)$ & $63(5.9)$ & $72(3.8)$ & \\
\hline Current drinker & $2,264(76.4)$ & $937(87.0)$ & $1,327(70.3)$ & \\
\hline \multicolumn{5}{|l|}{ Physical activity } \\
\hline Low & $2,209(74.5)$ & $828(77.0)$ & $1,381(73.2)$ & \multirow[t]{3}{*}{0.001} \\
\hline Moderate & $432(14.6)$ & $122(11.3)$ & $310(16.4)$ & \\
\hline High & $322(10.9)$ & $126(11.7)$ & $196(10.4)$ & \\
\hline \multicolumn{5}{|l|}{ Sleep duration (hr) } \\
\hline Q1 (<6.8) & $1,273(43.0)$ & $436(40.5)$ & $837(44.4)$ & \multirow[t]{4}{*}{0.052} \\
\hline Q2 (6.8-7.7) & $988(33.3)$ & $389(36.1)$ & $599(31.7)$ & \\
\hline Q3 (7.8-8.7) & $557(18.8)$ & $205(19.1)$ & 352 (18.7) & \\
\hline Q4 ( $\geq 8.8)$ & $145(4.9)$ & $46(4.3)$ & $99(5.2)$ & \\
\hline Energy intake (kcal/d) & $2,304.2 \pm 880.2$ & $2,737.1 \pm 984.3$ & $2,057.4 \pm 704.6$ & $<0.001$ \\
\hline Carbohydrate intake (\% energy) & $16.3 \pm 2.1$ & $15.7 \pm 2.4$ & $16.7 \pm 1.9$ & $<0.001$ \\
\hline Protein intake (\% energy) & $3.3 \pm 0.6$ & $3.1 \pm 0.5$ & $3.3 \pm 0.6$ & $<0.001$ \\
\hline Fat intake (\% energy) & $1.9 \pm 0.6$ & $1.9 \pm 0.6$ & $2.0 \pm 0.6$ & $<0.001$ \\
\hline Beck Depression Index & $9.6 \pm 7.1$ & $8.2 \pm 6.4$ & $10.5 \pm 7.4$ & $<0.001$ \\
\hline \multicolumn{5}{|l|}{ Lipid profile (mg/dL) } \\
\hline Total cholesterol & $203.6 \pm 33.8$ & $200.4 \pm 32.8$ & $205.3 \pm 34.2$ & $<0.001$ \\
\hline HDL-cholesterol & $58.3 \pm 14.8$ & $51.7 \pm 12.7$ & $62.1 \pm 14.6$ & $<0.001$ \\
\hline LDL-cholesterol & $119.2 \pm 31.9$ & $117.3 \pm 32.4$ & $120.3 \pm 31.6$ & 0.014 \\
\hline Triglyceride & $127.0 \pm 86.9$ & $156.0 \pm 111.3$ & $110.5 \pm 63.8$ & $<0.001$ \\
\hline \multicolumn{5}{|l|}{ Stressful life events } \\
\hline Total no. of life events & $3[1-5]$ & $3[1-5]$ & $2[1-5]$ & 0.150 \\
\hline Life events with positive effects & $1[1-2]$ & $1[1-2]$ & $1[1-2]$ & 0.389 \\
\hline Life events with negative effects & $1[1-3]$ & $1[1-3]$ & $1[1-2]$ & 0.430 \\
\hline
\end{tabular}

Values are presented as mean \pm standard deviation, median [interquartile range], or number (\%). $\mathrm{HDL}$, high-density lipoprotein; LDL, low-density lipoprotein.

'Independent t-test, chi-square test, or a non-parametric test (Mann-Whitney U test). 
Anni NS et al. : Stressful life events and triglycerides

Table 2. Associations between stressful life events and lipid levels in men and women (both pre- and post-menopause) ${ }^{1}$

\begin{tabular}{|c|c|c|c|c|}
\hline Stressful life events & $\mathrm{n}(\%)$ & Hypertriglyceridemia & Hypo-HDL-cholesterolemia & Hyper-LDL-cholesterolemia \\
\hline \multicolumn{5}{|l|}{ Men $(n=1,076)$} \\
\hline \multicolumn{5}{|c|}{ Total no. of life events } \\
\hline No & $171(15.9)$ & 1.00 (reference) & 1.00 (reference) & 1.00 (reference) \\
\hline Yes & $905(84.1)$ & $1.33(0.91,1.94)$ & $0.74(0.45,1.21)$ & $0.85(0.48,1.50)$ \\
\hline \multicolumn{5}{|c|}{ Life events with positive effects } \\
\hline No & $514(47.8)$ & 1.00 (reference) & 1.00 (reference) & 1.00 (reference) \\
\hline Yes & $562(52.2)$ & $1.16(0.89,1.53)$ & $0.83(0.59,1.17)$ & $0.77(0.50,1.17)$ \\
\hline \multicolumn{5}{|c|}{ Life events with negative effects } \\
\hline No & $375(34.9)$ & 1.00 (reference) & 1.00 (reference) & 1.00 (reference) \\
\hline Yes & $701(65.1)$ & $1.29(1.07,1.72)^{*}$ & $0.81(0.57,1.17)$ & $1.23(0.78,1.94)$ \\
\hline \multicolumn{5}{|l|}{ Women $(n=1,887)$} \\
\hline \multicolumn{5}{|c|}{ Total no. of life events } \\
\hline No & $315(16.7)$ & 1.00 (reference) & 1.00 (reference) & 1.00 (reference) \\
\hline Yes & $1,572(83.3)$ & $1.24(0.87,1.76)$ & $1.33(0.72,2.47)$ & $1.32(0.87,2.01)$ \\
\hline \multicolumn{5}{|c|}{ Life events with positive effects } \\
\hline No & $925(49.0)$ & 1.00 (reference) & 1.00 (reference) & 1.00 (reference) \\
\hline Yes & $962(51.0)$ & $1.47(1.14,1.89)^{*}$ & $0.92(0.56,1.51)$ & $1.12(0.84,1.49)$ \\
\hline \multicolumn{5}{|c|}{ Life events with negative effects } \\
\hline No & $674(35.7)$ & 1.00 (reference) & 1.00 (reference) & 1.00 (reference) \\
\hline Yes & $1,213(64.3)$ & $1.15(0.88,1.50)$ & $1.52(0.92,2.50)$ & $1.10(0.81,1.49)$ \\
\hline
\end{tabular}

Values are presented as odds ratio (95\% confidence interval).

$\mathrm{HDL}$, high-density lipoprotein; LDL, low-density lipoprotein.

${ }^{1}$ Results were derived from binary logistic regression models; Adjusted for age, body mass index, waist circumference, systolic blood pressure, diastolic blood pressure, education, marriage, smoking, drinking, physical activity, total energy, carbohydrate intake, fat intake, and depression score. ${ }^{*} \mathrm{p}<0.05$.

Table 3. Associations between stressful life events and hypertriglyceridemia in men and women divided into subgroups ${ }^{1}$

\begin{tabular}{|c|c|c|c|c|c|c|}
\hline Stressful life events & Total, $\mathrm{n}$ & $\begin{array}{c}\text { With } \\
\text { hypertriglyceridemia, } \\
\mathrm{n}(\%)\end{array}$ & Unadjusted & p-value & Adjusted $^{2}$ & $\mathrm{p}$-value \\
\hline \multicolumn{7}{|l|}{ Men $(n=1,076)$} \\
\hline \multicolumn{7}{|c|}{ Total no. of life events (events) } \\
\hline 0 & 171 & $59(34.5)$ & 1.00 (reference) & & 1.00 (reference) & \\
\hline 1 & 162 & $64(39.5)$ & $1.24(0.79,1.94)$ & 0.345 & $1.34(0.82,2.18)$ & 0.246 \\
\hline $2-3$ & 509 & $193(37.9)$ & $1.16(0.81,1.67)$ & 0.424 & $1.28(0.85,1.89)$ & 0.236 \\
\hline$\geq 4$ & 234 & $97(41.5)$ & $1.34(0.89,2.02)$ & 0.156 & $1.50(0.96,2.35)$ & 0.075 \\
\hline Per 1 & 1,076 & $413(38.4)$ & $1.04(1.00,1.08)$ & 0.044 & $1.05(1.01,1.09)$ & 0.023 \\
\hline \multicolumn{7}{|c|}{ Life events with positive effects (events) } \\
\hline 0 & 514 & $191(37.2)$ & 1.00 (reference) & & 1.00 (reference) & \\
\hline 1 & 257 & $103(40.1)$ & $1.13(0.83,1.54)$ & 0.432 & $1.30(0.93,1.83)$ & 0.122 \\
\hline $2-3$ & 281 & $106(37.7)$ & $1.02(0.76,1.38)$ & 0.875 & $0.99(0.71,1.37)$ & 0.938 \\
\hline$\geq 4$ & 24 & $13(54.2)$ & $1.99(0.88,4.55)$ & 0.099 & $2.57(1.02,6.46)$ & 0.045 \\
\hline Per 1 & 1,076 & $413(38.4)$ & $1.06(0.98,1.15)$ & 0.134 & $1.07(0.98,1.17)$ & 0.123 \\
\hline \multicolumn{7}{|c|}{ Sum of positive effect scores } \\
\hline 0 & 514 & $191(37.2)$ & 1.00 (reference) & & 1.00 (reference) & \\
\hline 1 & 137 & $55(40.1)$ & $1.13(0.77,1.67)$ & 0.522 & $1.44(0.95,2.19)$ & 0.086 \\
\hline $2-3$ & 303 & $118(38.9)$ & $1.08(0.81,1.45)$ & 0.611 & $1.07(0.78,1.48)$ & 0.672 \\
\hline$\geq 4$ & 122 & $49(40.2)$ & $1.14(0.76,1.70)$ & 0.538 & $1.13(0.73,1.75)$ & 0.578 \\
\hline Per 1 point & 1,076 & $413(38.4)$ & $1.02(0.98,1.06)$ & 0.401 & $1.02(0.98,1.07)$ & 0.340 \\
\hline
\end{tabular}


Table 3. Continued

\begin{tabular}{|c|c|c|c|c|c|c|}
\hline Stressful life events & Total, $\mathrm{n}$ & $\begin{array}{c}\text { With } \\
\text { hypertriglyceridemia, } \\
\mathrm{n}(\%)\end{array}$ & Unadjusted & p-value & Adjusted $^{2}$ & $p$-value \\
\hline \multicolumn{7}{|c|}{ Life events with negative effects (events) } \\
\hline 0 & 375 & $133(35.5)$ & 1.00 (reference) & & 1.00 (reference) & \\
\hline 1 & 240 & $100(41.7)$ & $1.30(0.93,1.81)$ & 0.122 & $1.43(0.99,2.05)$ & 0.052 \\
\hline $2-3$ & 384 & $141(36.7)$ & $1.06(0.79,1.42)$ & 0.725 & $1.13(0.82,1.56)$ & 0.453 \\
\hline$\geq 4$ & 77 & $39(50.6)$ & $1.87(1.14,3.06)$ & 0.013 & $1.99(1.16,3.41)$ & 0.012 \\
\hline Per 1 & 1,076 & $413(38.4)$ & $1.06(0.99,1.11)$ & 0.054 & $1.07(1.01,1.13)$ & 0.034 \\
\hline \multicolumn{7}{|c|}{ Sum of negative effect scores } \\
\hline 0 & 375 & $133(35.5)$ & 1.00 (reference) & & 1.00 (reference) & \\
\hline 1 & 172 & $66(38.4)$ & $1.13(0.78,1.65)$ & 0.512 & $1.16(0.77,1.74)$ & 0.476 \\
\hline $2-3$ & 362 & $136(37.6)$ & $1.09(0.81,1.48)$ & 0.553 & $1.22(0.88,1.69)$ & 0.234 \\
\hline$\geq 4$ & 167 & $78(46.7)$ & $1.60(1.10,2.31)$ & 0.014 & $1.71(1.11,2.55)$ & 0.008 \\
\hline Per 1 point & 1,076 & $413(38.4)$ & $1.03(0.99,1.06)$ & 0.071 & $1.04(1.01,1.08)$ & 0.028 \\
\hline \multicolumn{7}{|l|}{ Women $(n=1,887)$} \\
\hline \multicolumn{7}{|c|}{ Total no. of life events (events) } \\
\hline 0 & 315 & $46(14.6)$ & 1.00 (reference) & & 1.00 (reference) & \\
\hline 1 & 334 & $55(16.5)$ & $1.15(0.75,1.77)$ & 0.513 & $1.09(0.70,1.70)$ & 0.706 \\
\hline $2-3$ & 864 & $151(17.5)$ & $1.24(0.87,1.77)$ & 0.242 & $1.25(0.86,1.82)$ & 0.238 \\
\hline$\geq 4$ & 374 & $69(18.4)$ & $1.32(0.88,1.97)$ & 0.178 & $1.41(0.92,2.16)$ & 0.116 \\
\hline Per 1 & 1,887 & $321(17.0)$ & $1.01(0.97,1.05)$ & 0.812 & $1.02(0.97,1.06)$ & 0.506 \\
\hline \multicolumn{7}{|c|}{ Life events with positive effects (events) } \\
\hline 0 & 925 & $138(14.9)$ & 1.00 (reference) & & 1.00 (reference) & \\
\hline 1 & 451 & $91(20.2)$ & $1.44(1.08,1.93)$ & 0.014 & $1.55(1.14,2.10)$ & 0.005 \\
\hline $2-3$ & 475 & $87(18.3)$ & $1.28(0.95,1.72)$ & 0.102 & $1.41(1.04,1.93)$ & 0.029 \\
\hline$\geq 4$ & 36 & $5(13.9)$ & $0.92(0.35,2.41)$ & 0.865 & $1.01(0.36,2.73)$ & 0.976 \\
\hline Per 1 & 1,887 & $321(17.0)$ & $1.02(0.94,1.10)$ & 0.748 & $1.03(0.95,1.12)$ & 0.431 \\
\hline \multicolumn{7}{|c|}{ Sum of positive effect score } \\
\hline 0 & 925 & $138(14.9)$ & 1.00 (reference) & & 1.00 (reference) & \\
\hline 1 & 231 & $52(22.5)$ & $1.66(1.16,2.37)$ & 0.006 & $1.70(1.17,2.47)$ & 0.006 \\
\hline $2-3$ & 515 & $95(18.4)$ & $1.29(0.97,1.72)$ & 0.082 & $1.47(1.09,1.99)$ & 0.013 \\
\hline$\geq 4$ & 216 & $36(16.7)$ & $1.14(0.76,1.70)$ & 0.520 & $1.22(0.80,1.85)$ & 0.363 \\
\hline Per 1 point & 1,887 & $321(17.0)$ & $1.08(0.97,1.20)$ & 0.162 & $1.12(1.01,1.26)$ & 0.044 \\
\hline \multicolumn{7}{|c|}{ Life events with negative effects (events) } \\
\hline 0 & 674 & $106(15.7)$ & 1.00 (reference) & & 1.00 (reference) & \\
\hline 1 & 421 & $79(18.8)$ & $1.24(0.90,1.71)$ & 0.192 & $1.15(0.82,1.60)$ & 0.427 \\
\hline $2-3$ & 689 & $121(18.6)$ & $1.14(0.86,1.52)$ & 0.364 & $1.20(0.89,1.62)$ & 0.233 \\
\hline$\geq 4$ & 103 & $15(14.6)$ & $0.91(0.51,1.64)$ & 0.762 & $0.92(0.52,1.89)$ & 0.777 \\
\hline Per 1 & 1,887 & $321(17.0)$ & $1.01(0.99,1.06)$ & 0.877 & $1.01(0.94,1.07)$ & 0.916 \\
\hline \multicolumn{7}{|c|}{ Sum of negative effect score } \\
\hline 0 & 674 & $106(15.7)$ & 1.00 (reference) & & 1.00 (reference) & \\
\hline 1 & 304 & $62(20.4)$ & $1.37(0.97,1.94)$ & 0.074 & $1.23(0.85,1.77)$ & 0.269 \\
\hline $2-3$ & 620 & $102(16.5)$ & $1.06(0.78,1.42)$ & 0.723 & $1.13(0.82,1.54)$ & 0.459 \\
\hline$\geq 4$ & 289 & $51(17.6)$ & $1.15(0.80,1.66)$ & 0.460 & $1.10(0.75,1.62)$ & 0.619 \\
\hline Per 1 point & 1,887 & $321(17.0)$ & $1.03(0.92,1.15)$ & 0.623 & $1.04(0.92,1.16)$ & 0.550 \\
\hline
\end{tabular}

Values are presented as odds ratio (95\% confidence interval). ${ }^{1}$ Results were derived from binary logistic regression models.

${ }^{2}$ Adjusted for age, body mass index, waist circumference, systolic blood pressure, diastolic blood pressure, education, marriage, smoking, drinking, physical activity, total energy, carbohydrate intake, fat intake, and depression score. 
Table 4. Association between stressful life events and triglyceride levels in men and women ${ }^{1}$

\begin{tabular}{lcccc}
\hline Stressful life events & Men & p-value & Women & p-value \\
\hline Total no. of life events & $3.333(1.381,5.285)$ & 0.001 & $0.451(-0.471,1.373)$ & 0.338 \\
Life events with positive effects & $5.557(1.499,9.616)$ & 0.007 & $0.766(-1.059,2.592)$ & 0.410 \\
Sum of positive effect score & $2.229(0.103,4.456)$ & 0.041 & $0.452(-0.506,1.410)$ & 0.355 \\
Life events with negative effects & $3.286(0.491,6.081)$ & 0.021 & $0.126(-1.203,1.456)$ & 0.852 \\
Sum of negative effect score & $1.388(-0.275,3.015)$ & 0.093 & $0.053(-0.726,0.832)$ & 0.133 \\
\hline
\end{tabular}

Values are presented as $\beta$ ( $95 \%$ confidence interval).

${ }^{1}$ Results were derived from multiple linear regression models; Adjusted for age, body mass index, waist circumference, systolic blood pressure, diastolic blood pressure, education, marriage, smoking, drinking, physical activity, total energy, carbohydrate intake, fat intake, and depression score.

\section{DISCUSSION}

Our study results demonstrate that experiencing SLEs was associated with elevated blood triglyceride levels and the presence of hypertriglyceridemia, especially among adult men. Both positive and negative SLEs may increase blood triglyceride levels, although the association was clearer for SLEs with negative effects. However, women were more likely to show an association between SLEs with positive effects and hypertriglyceridemia. Previous studies have considered workplace stress or childhood trauma as triggers of increasing serum triglyceride levels $[9,10,22]$. However, in our study, we found that SLEs with either positive or negative impacts had a positive association with increased triglyceride levels. In a Finnish cohort study of 3,407 participants (1,618 men and 1,789 women), metabolic syndrome was associated with SLEs related to work or finances, and the risk was further increased with having at least 3 SLEs in any of the life-related domains assessed. However, the change in serum triglyceride levels did not show any gender stratification [23]. A Dutch cohort study of a middleaged and elderly population $(n=1,099)$ reported a positive association between the number of SLEs (especially financial) and the risk of developing metabolic syndrome (OR, 2.25; 95\% CI, 1.17 to 4.33) [24].

The association between SLEs and hypertriglyceridemia can be explained by several possible mechanisms. One of the plausible mechanisms is a change in health behaviors after experiencing SLEs. Studies from Iran have demonstrated that a stressor in the past 6-12 months resulted in elevated triglyceride levels [7,25]. Stressors can alter health behaviors such as smoking, drinking, exercise, sleeping, and eating habits, which result in the elevation of blood triglycerides [26,27]. A cohort study conducted among 2,850 Dutch participants showed that the severity of anxiety or depression was adversely associated with smoking, which might detrimentally influence triglyceride levels [28]. Although we adjusted for lifestyle factors, we cannot exclude the effect of behavior changes.

Stressors stimulate the activity of the HPA axis, resulting in increased cortisol levels, which can elevate circulating triglyceride levels [29]. However, this response to stress is determined by the number of SLEs, the impact of an event, and the ability of an individual to adapt [30]. For example, marriage can be considered a positive event or negative event and can act as a stressor, depending on an individual's sense of control, the stability of his or her status, or way of adaptation to life changes [31]. This offers a possible explanation for why men and women were influenced by life changes in different ways in our study. The way of adaptation to life changes might play a pivotal role in the change of serum triglyceride levels in response to SLEs with positive and negative effects in men and women. In our study, we considered the number of events instead of scrutinizing the impact of each event on serum triglyceride levels separately, as the events varied in type. We also took into account the score of each event, both positive and negative. There are no standard objective weights for SLEs; therefore, we used scores as a measure of the direction and strength of each event. We found a dose-response association between hypertriglyceridemia and an increase of SLE, especially for SLEs with negative effects and the score in men, whereas women did not show any significant association. In addition, graded associations between hypertriglyceridemia and SLEs with both positive and negative effects in men were observed, when the number of events was $\geq 4$. However, we did not find a significant association between the scores of SLEs with positive effects and hypertriglyceridemia when the scores were $\geq 4$. A likely explanation for this finding may be that the single event with the highest impact can affect an individual especially strongly; nevertheless, the frequency of events, regardless of the magnitude of their impact, also matters.

In our study, the relationship between SLEs and triglyceride levels was more prominent in men than in women. It has been reported that the activity of the HPA axis in response to stress is greater in men than in women [32]. Further, the metabolic clearance rate of fatty acids is higher in women than in men [33]. Although this cannot fully explain our results, we can hypothesize that physiological mechanisms may have contributed to the finding that serum triglyceride levels were higher in man participants than in woman participants in response to SLEs.

To the best of our knowledge, this study was the first to elucidate the association between SLEs and hypertriglyceridemia in a middle-aged Korean population. To minimize the possibility of distortion caused by prevalent diseases or medication use, we excluded people with major cardiovascular disease or cancer and people taking any lipid-controlling medications or other treatments that might influence serum triglyceride levels. However, 
the study results may also have implications for dyslipidemia patients taking lipid-lowering agents or patients with diabetes on medication for glycemic control. Prior studies have reported that stress stimulates several hormones like norepinephrine, epinephrine, cortisol, $\beta$-endorphin, and growth hormone, which can affect glucose homeostasis in healthy people and in patients with diabetes [34,35]. Moreover, hypertriglyceridemia has adverse effects on insulin sensitivity and islet beta-cell function, which might affect glycemic control [36]. Our study illustrated that SLEs can play a role in increasing serum triglyceride levels; therefore, more attention should be paid to the proper management of stressors in dyslipidemia patients taking lipid-lowering drugs and patients with diabetes. Our study measured 50 types of potential SLE experiences with their impact score, while most previous studies only included a limited number of events or evaluated only the presence or absence of SLEs.

There are several limitations of the current study. First, this is a cross-sectional analysis of baseline data of the CMERC cohort study; thus, the temporal relationship between SLEs and serum triglyceride levels could not be established. Second, data were collected by in-person interviews; measurement errors and recall bias may have affected the accuracy of the data. Third, although adjustment was conducted for potential confounders, the possibility of residual confounding remains. Fourth, we cannot rule out the possibility that the observed association of SLEs and hypertriglyceridemia in men reflects type I error due to multiple tests. Considering this limitation, we interpreted our findings as exploratory results, and further replication studies are needed for confirmation.

In conclusion, our study shows that SLEs might have adverse effects on triglyceride metabolism, especially in men. Considering the high prevalence of hypertriglyceridemia among middle-aged Korean men, adequate management of stressors in daily life might be a challenge for cardiovascular disease prevention. Further research is needed on gender differences in the association between SLEs and triglyceride levels.

\section{SUPPLEMENTARY MATERIALS}

Supplementary materials are available at http://www.e-epih. org/.

\section{CONFLICT OF INTEREST}

The authors have no conflicts of interest to declare for this study.

\section{FUNDING}

This work was supported by a grant of the Korea Health Technology R\&D Project through the Korea Health Industry Development Institute (KHIDI) funded by the Ministry of Health and Welfare, Republic of Korea (grant No. HI13C0715), and the Basic
Science Research Program through the National Research Foundation of Korea (NRF) funded by the Ministry of Science and ICT (NRF-2019R1A4A1028155).

\section{ACKNOWLEDGEMENTS}

The authors appreciate all cohort members who participated voluntarily in the study. We are also grateful to the members of the research staff for their passion.

\section{AUTHOR CONTRIBUTIONS}

Conceptualization: NSA, HCK. Data curation: JSS, GBL. Formal analysis: NSA. Funding acquisition: HCK. Methodology: NSA, SJJ, JSS, HCK. Project administration: JSS, YWJ, HCK. Visualization: NSA. Writing - original draft: NSA, HCK. Writing review \& editing: SJJ, JSS, YWJ, GBL, HCK.

\section{ORCID}

Naharin Sultana Anni: https://orcid.org/0000-0001-9523-5352; Sun Jae Jung: https://orcid.org/0000-0002-5194-7339; Jee-Seon Shim: https://orcid.org/0000-0002-8671-3153; Yong Woo Jeon: https://orcid.org/0000-0002-0659-4159; Ga Bin Lee: https://orcid. org/0000-0001-6574-1414; Hyeon Chang Kim: https://orcid.org/ 0000-0001-7867-1240

\section{REFERENCES}

1. Generoso G, Janovsky CC, Bittencourt MS. Triglycerides and triglyceride-rich lipoproteins in the development and progression of atherosclerosis. Curr Opin Endocrinol Diabetes Obes 2019;26: 109-116.

2. Nordestgaard BG, Varbo A. Triglycerides and cardiovascular disease. Lancet 2014;384:626-635.

3. Austin MA, Hokanson JE, Edwards KL. Hypertriglyceridemia as a cardiovascular risk factor. Am J Cardiol 1998;81:7B-12B.

4. Yaribeygi H, Panahi Y, Sahraei H, Johnston TP, Sahebkar A. The impact of stress on body function: a review. EXCLI J 2017;16:10571072 .

5. Kivimäki M, Steptoe A. Effects of stress on the development and progression of cardiovascular disease. Nat Rev Cardiol 2018;15: 215-229.

6. Björntorp P. Visceral fat accumulation: the missing link between psychosocial factors and cardiovascular disease? J Intern Med 1991;230:195-201.

7. Assadi SN. What are the effects of psychological stress and physical work on blood lipid profiles? Medicine (Baltimore) 2017;96: e6816.

8. Garbarino S, Magnavita N. Work stress and metabolic syndrome in police officers. A prospective study. PLoS One 2015;10:e0144318.

9. Rose G, Kumlin L, Dimberg L, Bengtsson C, Orth-Gomer K, Cai $\mathrm{X}$. Work-related life events, psychological well-being and cardio- 
vascular risk factors in male Swedish automotive workers. Occup Med (Lond) 2006;56:386-392.

10. Park JH, Jung SJ, Jung Y, Ahn SV, Lee E, Kim HC. Association between the change of total cholesterol during adolescence and depressive symptoms in early adulthood. Eur Child Adolesc Psychiatry 2021;30:261-269.

11. Shim JS, Song BM, Lee JH, Lee SW, Park JH, Choi DP, et al. Cardiovascular and Metabolic Diseases Etiology Research Center (CMERC) cohort: study protocol and results of the first 3 years of enrollment. Epidemiol Health 2017;39:e2017016.

12. Sarason IG, Johnson JH, Siegel JM. Assessing the impact of life changes: development of the Life Experiences Survey. J Consult Clin Psychol 1978;46:932-946.

13. Lee Y. Association between depression and attribution style, life event, event attribution and hopelessness: structural equation model analysis [dissertation]. Seoul: Seoul National University; 1993 (Korean).

14. Kushner PA, Cobble ME. Hypertriglyceridemia: the importance of identifying patients at risk. Postgrad Med 2016;128:848-858.

15. Expert Panel on Detection, Evaluation, and Treatment of High Blood Cholesterol in Adults. Executive summary of the third report of the National Cholesterol Education Program (NCEP) Expert Panel on Detection, Evaluation, and Treatment of High Blood Cholesterol in Adults (Adult Treatment Panel III). JAMA 2001;285: 2486-2497.

16. Kim SK, Kim HC, Shim JS, Kim DJ. Effects of cigarette smoking on blood lipids in Korean men: Cardiovascular and Metabolic Diseases Etiology Research Center cohort. Korean J Intern Med 2020;35:369-382.

17. Chun MY. Validity and reliability of Korean version of international physical activity questionnaire short form in the elderly. Korean J Fam Med 2012;33:144-151.

18. International Physical Activity Questionnaires (IPAQ) Research Committee. Guidelines for data processing and analysis of the IPAQ-short and long forms; 2019 [cited 2019 Dec 31]. Available from: http://www.ipaq.ki.se.

19. Lim SY, Kim SJ. Validation of a short Korean version of the UPPSP Impulsive Behavior Scale. Asia Pac Psychiatry 2018;10:e12318.

20. Yun SH, Shim JS, Kweon S, Oh K. Development of a food frequency questionnaire for the Korea National Health and Nutrition Examination Survey: data from the fourth Korea National Health and Nutrition Examination Survey (KNHANES IV). J Nutr Health 2013;46:186-196 (Korean).

21. Park JH, Lee MH, Shim JS, Choi DP, Song BM, Lee SW, et al. Effects of age, sex, and menopausal status on blood cholesterol profile in the Korean population. Korean Circ J 2015;45:141-148.

22. Péterfalvi Á, Németh N, Herczeg R, Tényi T, Miseta A, Czéh B, et al. Examining the influence of early life stress on serum lipid pro- files and cognitive functioning in depressed patients. Front Psychol 2019;10:1798.

23. Pyykkönen AJ, Räikkönen K, Tuomi T, Eriksson JG, Groop L, Isomaa B. Stressful life events and the metabolic syndrome: the prevalence, prediction and prevention of diabetes (PPP)-Botnia Study. Diabetes Care 2010;33:378-384.

24. Rutters F, Pilz S, Koopman AD, Rauh SP, Pouwer F, Stehouwer $\mathrm{CD}$, et al. Stressful life events and incident metabolic syndrome: the Hoorn study. Stress 2015;18:507-513.

25. Fakhari A, Ebrahimzadeh M, Shiva S, Fekrat S, Mohammadpoorasl A. Effect of mental stress on serum triglyceride level. Res J Biol Sci 2007;2:476-478.

26. Bachen EA, Muldoon MF, Matthews KA, Manuck SB. Effects of hemoconcentration and sympathetic activation on serum lipid responses to brief mental stress. Psychosom Med 2002;64:587594.

27. Brindley DN, Rolland Y. Possible connections between stress, diabetes, obesity, hypertension and altered lipoprotein metabolism that may result in atherosclerosis. Clin Sci (Lond) 1989;77:453461.

28. van Reedt Dortland AK, Vreeburg SA, Giltay EJ, Licht CM, Vogelzangs $\mathrm{N}$, van Veen $\mathrm{T}$, et al. The impact of stress systems and lifestyle on dyslipidemia and obesity in anxiety and depression. Psychoneuroendocrinology 2013;38:209-218.

29. Jacobson L. Hypothalamic-pituitary-adrenocortical axis: neuropsychiatric aspects. Compr Physiol 2014;4:715-738.

30. John OP, Gross JJ. Healthy and unhealthy emotion regulation: personality processes, individual differences, and life span development. J Pers 2004;72:1301-1333.

31. Holmes TH, Rahe RH. The social readjustment rating scale. J Psychosom Res 1967;11:213-218.

32. Verma R, Balhara YP, Gupta CS. Gender differences in stress response: role of developmental and biological determinants. Ind Psychiatry J 2011;20:4-10.

33. Sarac I, Backhouse K, Shojaee-Moradie F, Stolinski M, Robertson MD, Bell JD, et al. Gender differences in VLDL1 and VLDL2 triglyceride kinetics and fatty acid kinetics in obese postmenopausal women and obese men. J Clin Endocrinol Metab 2012;97: 2475-2481.

34. Surwit RS, Schneider MS. Role of stress in the etiology and treatment of diabetes mellitus. Psychosom Med 1993;55:380-393.

35. Dungan KM, Braithwaite SS, Preiser JC. Stress hyperglycaemia. Lancet 2009;373:1798-1807.

36. Ma M, Liu H, Yu J, He S, Li P, Ma C, et al. Triglyceride is independently correlated with insulin resistance and islet beta cell function: a study in population with different glucose and lipid metabolism states. Lipids Health Dis 2020;19:121. 